\title{
LAS EDUCACIONES AFRICANAS A LO LARGO DE LOS SIGLOS: UNA NAVEGACIÓN ENTRE VARIOS SISTEMAS EDUCATIVOS
}

\section{African education over the centuries: a navigation between various educational systems}

\author{
Eugénie EYEANG \\ CRAAL, École Normale Supérieure (Libreville, Gabon) \\ Correo-e: eyeangeugenie@gmail.com \\ Recibido: Io de abril de 2020 \\ Envío a informantes: 13 de abril de 2020 \\ Aceptación definitiva: 9 de mayo de 2020
}

Resumen: Ni África es un continente uniforme (podríamos hablar de varias Áfricas), ni tampoco se puede hablar de una sola educación africana, por lo que hemos de hablar en plural. Más aún si nos referimos a la enorme diversidad de pueblos, lenguas y culturas que pueblan el continente de norte a sur y de este a oeste. También parece obvio hablar de diversidad y en plural cuando nos referimos a los sistemas educativos actuales de las diferentes naciones africanas, que construyen sus modelos educativos propios a partir de sus respectivas independencias en el corazón del siglo xx, pero tomando en consideración muchas de las tradiciones africanas ancestrales de los pueblos originarios. En esta confluencia de situaciones se encierra la clave interpretativa del ser educativo del continente africano actual, que posee riquísimas y tradiciones ancestrales, incluso con culturas escritas sincrónicas a las griegas, las judeocristianas primitivas y árabes, y no solo orales, rompiendo así alguno de los tópicos imperantes sobre la ausencia de la cultura escrita entre los africanos. Un análisis comparado de varias muestras y ejemplos nos conduce a una interpretación menos lineal y tradicional de los modelos educativos africanos de nuestro tiempo.

Palabras Clave: África, educación; sistemas educativos; culturas africanas originarias.

Aвstract: Neither is Africa a uniform continent (we could speak of several Africans), nor can we speak of a single African education, so we have to speak in plural. 
Even more so if we refer to the enormous diversity of peoples, languages and cultures that populate the continent from north to south and from east to west. It also seems obvious to speak of diversity and plural when we refer to the current educational systems of the different African nations, which build their own educational models from their respective independence at the heart of the twentieth century, but taking into consideration many of the ancestral African traditions of the original peoples. In this confluence of situations is contained the interpretative key of the educational being of the present African continent, which possesses very rich and ancestral traditions, even with written cultures synchronous to the Greek, Judeo-Christian primitives and Arabs, and not just oral, thus breaking some of the prevailing clichés about the absence of written culture among Africans. A comparative analysis of several samples and examples leads to a less linear and traditional interpretation of the African educational models of our time.

KEY WORDs: Africa; education; education systems; native African cultures.

\section{Introducción}

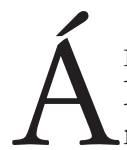

FRICA ES UN CONTINENTE COMPLEJO por esencia y variado por sus influencias. Desde tiempos remotos, el genio africano se ha manifestado en varios sectores de la vida social (Antonio Fernández, 2016). Cada país africano ha estado en contacto con más de una cultura exógena y concepción del mundo. En materia de educación, del norte al sur, del oeste al este, existen diversas formas africanas de educación, unas más tradicionales que otras. De una zona a otra, el enfoque educativo varía con una perspectiva endógena. Dentro de un mismo espacio geolingüístico y cultural, la práctica de la educación es común a los miembros de tal comunidad. Por eso, tenemos, hasta hoy, cierta homogeneidad en los principios de la educación en zonas de predominancia de los grandes imperios africanos: el Reino de Aksum (50937), el Imperio de Ghana (750-I068), el Reino de Benín (I440-I897), el Reino de Gabú o Senegambia (I532-I867), el Reino de Luba (I585-I885), el Imperio Etíope (II37-I974), etc. Unos datos históricos informan sobre la organización sociopolítica y económica de buena parte de África'. Notamos que:

A mediados del siglo vili encontramos ya el poderoso Estado de Ghana que controlaba el comercio entre las zonas productoras de oro del golfo de Guinea y las caravanas de camellos árabes. Durante los siglos XiI y xiII el Imperio de Malí es estado dominante del occidente africano. En el siglo xvi, el Estado musulmán del Imperio Songhaï es quien adquiere mayor control político y económico de esta zona. En Nigeria, el reino de Benín empezó a surgir en el duodécimo siglo. Había desarrollado una poderosa ciudad-Estado cuando los portugueses llegan en el siglo Xv. Lo mismo sucedió con Ife, el centro de los Yoruba en el sudoeste de Nigeria. En Zimbabwe, en el siglo x surge un importante centro comercial conocido como el Gran Zimbabwe,

Antiguos Estados africanos (cf. Historia de África): Hasta el siglo vi: Kerma, Egipto, Kush, Meroe, Aksum, Axum, Estados Nubios. Del siglo vir a xII: Ghana, Marruecos, Imperio Fatimi, Almoravide, Zanj Del siglo xiı a Xvi: Mali, Songhay, Estados Hausa, Kanem-Bornu, Benín, Yoruba, Kongo, Mapungubwe, Gran Zimbabwe, Monomotapa, Funj. Del siglo xvir a xix: Imperio Rozwi, Jollof, Mossi, Akan (Asante), Kuba, Luba, Lunda, Zulu, Bamum, Bunyoro, Buganda, Lozi o Barotse, Kwanyama, Fulani, Jukun, Nupe (cf. http://www.ikuska.com/Africa/Historia/estados/index.htm, consulta el 13.05.2018). 

EUGÉNIE EYEANG

muy activo en el comercio del oro que producían e intercambiaban con productos como vidrio y alfarería de China procedentes de la costa del Índico. Se mantuvo hasta mediados del siglo xv.

(http://www.ikuska.com/Africa/Historia/estados/index.htm).

Estos datos nos dan a entender que esas organizaciones estatales y espirituales no podían desarrollarse sin bases educativas de progreso para las poblaciones. El enfoque comunitario (Canales, 2007) en vigor en esas comunidades tiene establecida una particular para cada miembro. Es verdad que la educación en África se ha basado durante generaciones en la transmisión oral de conocimientos prácticos relacionados con las labores cotidianas, con la cosmovisión de cada pueblo, con la relación entre los seres humanos y la naturaleza que les rodeaba.

Ya con la llegada a África de los árabes en los siglos viI-IX y especialmente de los europeos en los siglos XVII-XviII, se impuso una educación muy distinta, basada en lenguas extranjeras, con las que se construían esquemas mentales ajenos y muy diferentes. Establecidos a imagen y semejanza de los países europeos, la mayoría de los sistemas educativos africanos son copia fiel y herencia del existente en las metrópolis europeas (Eyeang, 1997; Sanz Martín, 20II) en la época de la colonización, en la primera mitad del siglo $x x$.

A partir del siglo XIX, con la colonización y la penetración de paradigmas diferentes sobre el saber y la organización formal del aprendizaje, África navega entre varias percepciones del mundo. Está en una situación de deconstrucción y construcción/ re-construcción de los conocimientos. Por eso, muchos sistemas educativos africanos no consiguen proponer vías congruentes y pertinentes para la mejora de sus sociedades. El artículo de Pedro Alberto Sanz Martín (20II) que trata de la educación tradicional africana en la escuela actual en África subsahariana es muy interesante para el tema de la educación comparada. Da una visión global y positiva de la educación tradicional africana. Recalca los hechos históricos de la colonización en materia de educación reconociendo unos logros realizados antes del periodo colonial.

Aparte de Etiopía, también en otras zonas de África hubo contactos con la lectura y la escritura, especialmente con la lengua árabe. Destaca, sobre todo, la ciudad de Tombuctú, en el actual Estado de Malí, situada en la franja de contacto entre el África islámica y el África Subsahariana. En estas zonas de influencia islámica, ya antes de la colonización europea, existían las escuelas coránicas fuertemente vinculadas a la tradición religiosa y basadas en la difusión de valores religiosos, sociales y culturales islámicos, donde también se impartían conocimientos de Matemáticas, Física, Ciencias Naturales, Derecho, Historia y Literatura Arábiga (Sanz Martín, 20II: 58-59).

El objetivo de este trabajo es mostrar la necesidad de descubrir, estudiar y comparar el complejo entramado que representa en cada pueblo el proceso educativo ${ }^{2}$. Los fundamentos de la educación comparada nos sirven de marco de referencia (García Garrido, 199I). La metodología adoptada es la del análisis documental y de contenido a partir de entrevistas semidirectas dirigidas a profesionales de la enseñanza sobre las educaciones africanas y el rendimiento de los sistemas educativos actuales.

Algunos aspectos de este estudio aparecen en nuestro estudio, Eyeang, 20I7: 17-36. 
Los beneficios del estudio permiten ver cómo los sistemas educativos africanos, a partir de aspectos conceptuales y comparativos, podrán contribuir a su mejora. El método por excelencia utilizado está constituido por cuatro etapas o fases: descripción, interpretación, yuxtaposición y comparación.

\section{Visión de la educación tradicional africana a través de los siglos}

\section{I.I. Una visión fuera de los caminos trillados}

Pocos son los documentos que tratan de la educación africana antes de la colonización de manera favorable. Algunos estudios etnográficos, antropológicos o arqueológicos permiten ver los vestigios de esa educación y contradicen unos tópicos sobre esa educación. El artículo de Antonio Fernández (2016), que hemos revisado, presenta una visión de un África vanguardista antes de la llegada de los europeos a partir de diez hechos educativos. Retenemos aquí los más sobresalientes. Para él, la creencia de que la educación en la antigua África era oral y no escrita es más que un mito. Desde el año 300 antes de Cristo al 350 después de Cristo, la civilización Meroe (actual Sudán) desarrolló un sistema de escritura propio. Eso se hizo por la influencia árabe.

\section{Fotografía n. ${ }^{\circ}$ I: Manuscritos}

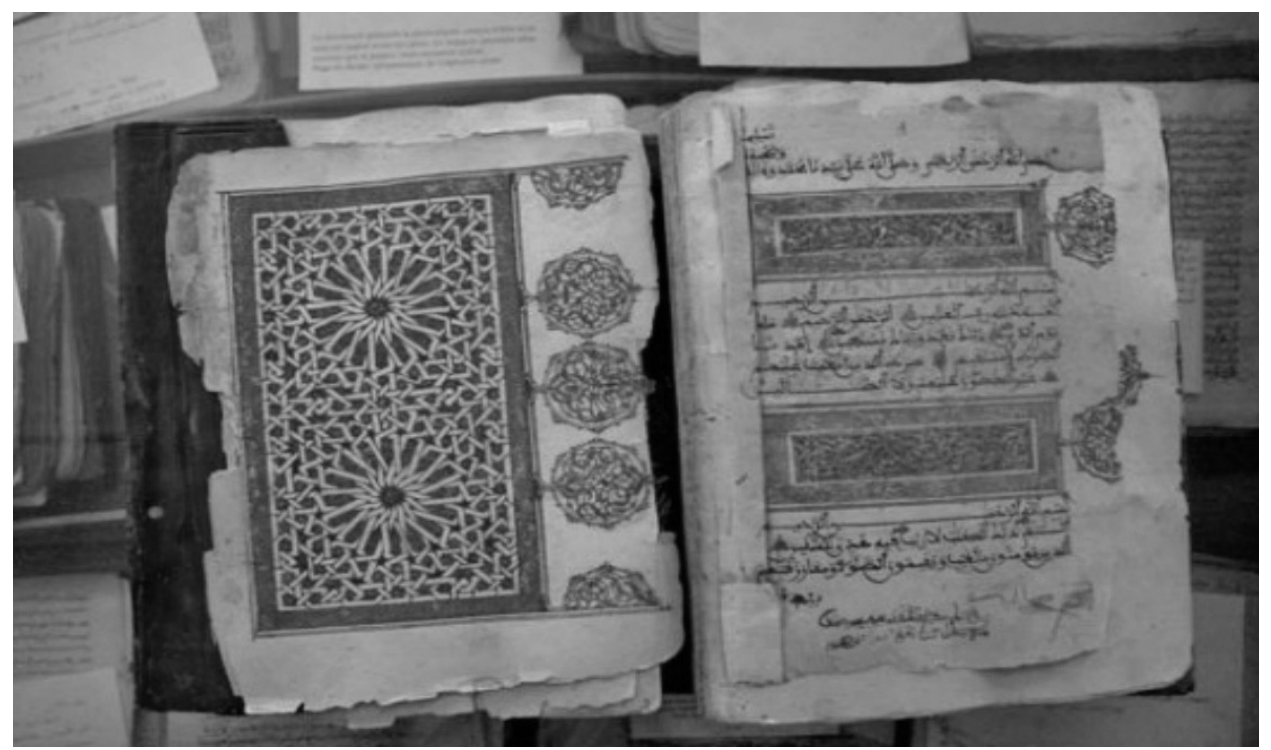

Fuente: Internet 2018.

África creó una de las universidades más antiguas del mundo. Fundada en el año 989 por el juez principal erudito de Tombuctú, Al-Qadi Aqib Ibn Mahmud Ibn Umar, la Mezquita Sankore o Universidad de Sankore es una de las más antiguas escuelas de educación superior en el mundo. Las zonas con mayor prestigio económico y cultural se recrean y permiten el desarrollo de varias actividades. Los individuos de Egipto y 

EUGÉNIE EYEANG

Nubia desarrollaron «alfabetización, núcleos urbanos y unas civilizaciones tecnológicamente avanzadas» siglos antes de que existieran Roma y Atenas. Además, muchas personas viajaron desde todas las partes del mundo para estudiar en las universidades de Tombuctú, también en los imperios de Malí y Songhai. Se ha podido comprobar que eruditos europeos venerados, como Herodoto, viajaron a África para aprender y obtener información (Antonio Fernández, 2016).

\section{Fotografía n. ${ }^{\circ}$ 2: Universidad de Sankore}

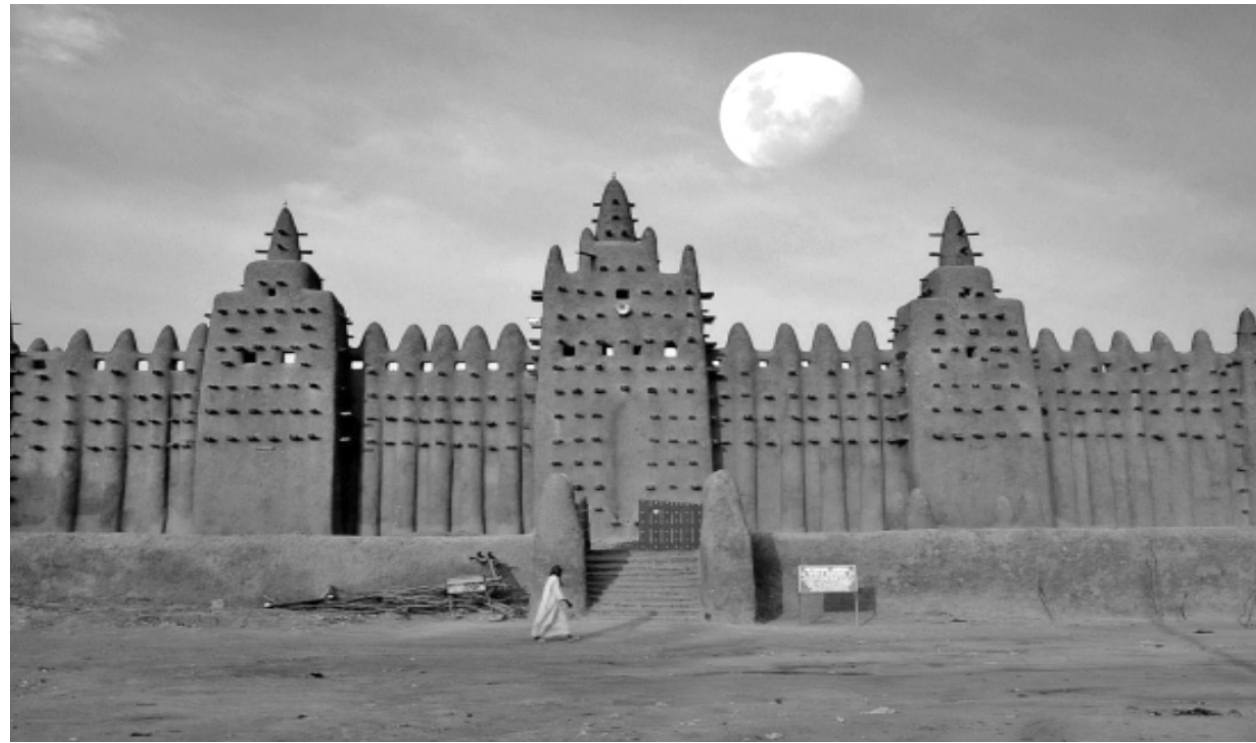

Fuente: Internet 2018.

Con esos datos, vemos que África era un centro educativo de mayor índole que ayudó a otros pueblos a obtener conocimientos y formarse. La pregunta que aflora, obviamente, es que la mayoría de los países africanos no sacaron provecho de esos avances. ¿Cuál fue el elemento perturbador que impidió la eclosión de la inteligencia africana? No contestaremos a esa pregunta en este trabajo, pero este aspecto merece unas reflexiones para entender cómo los primeros se volvieron los últimos en materia de educación.

\section{I.2. Concepciones etológicas de la educación africana}

En África la educación es considerada como la clave que facilita la integración en la sociedad. Da valores y ofrece principios a aplicar en cada situación. La educación tenía un carácter colectivo pronunciado, una globalidad a nivel de agentes. Todo el tejido social sirve como marco de acción. La educación es global e integrada en la vida. La educación tradicional se realiza en todas partes y en todas ocasiones, en el contexto habitual de trabajo y ocio (Wilfried Koikson, 20I8). 


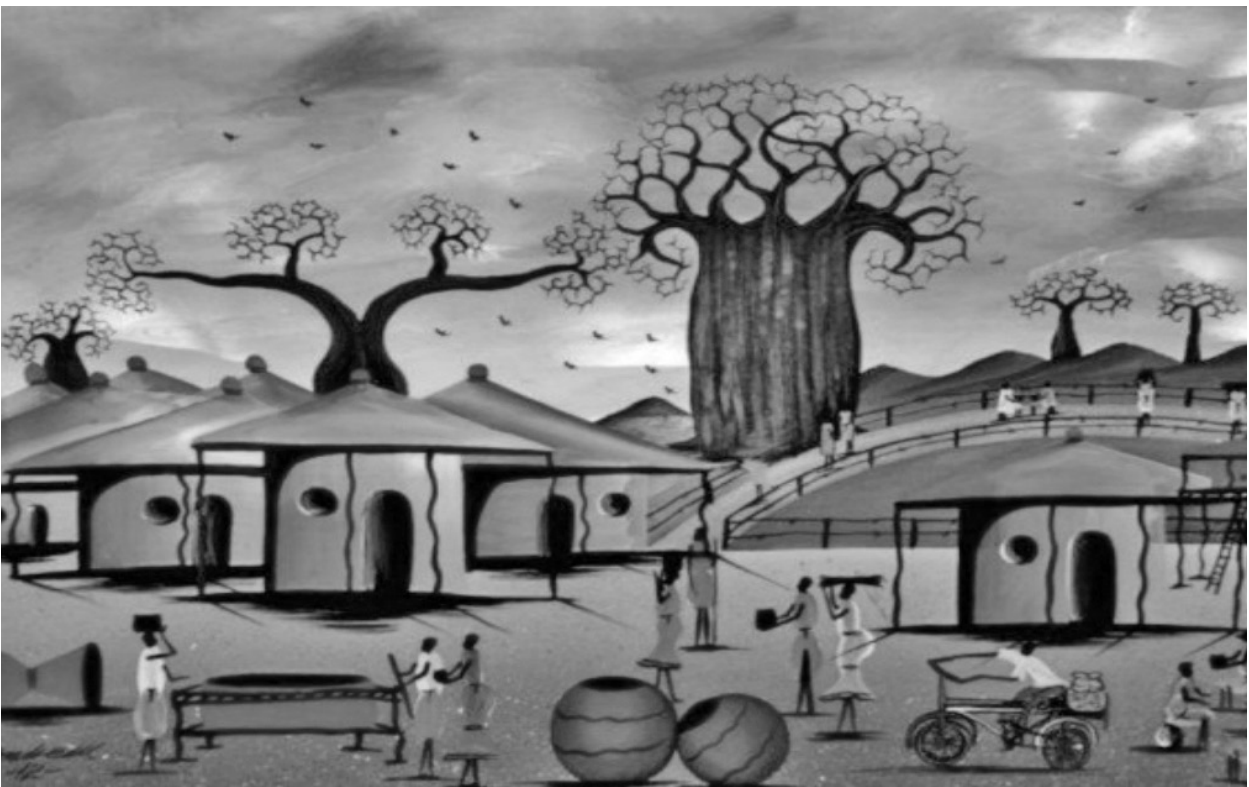

Fuente: Internet 2018.

Tradicionalmente, todo el mundo estaba involucrado en el proceso educativo. El principal objetivo de la educación tradicional en África era producir un individuo completo; uno que fuera culto, respetuoso, integrado, sensible y receptivo a las necesidades de la familia y los vecinos.

Toda una comunidad, un pueblo, actúa para criar a un niño. En lo que respecta a la educación de África antes de la llegada de los europeos, cada generación estaba involucrada. La educación indígena en África se caracterizó por el «proceso de transmitir entre los miembros de la tribu y de una generación a otra el conocimiento heredado, habilidades, tradiciones culturales, normas y valores de la tribu» (Antonio Fernández, 2016). Casi todos los africanos hasta los años 1950-70 han recibido formación e instrucción por la tarde alrededor del fuego de la tribu, cuando las personas desean recibir calor.

La función principal del cuentista (narrador) en las sociedades tradicionales consistía, pues, en promover la incuestionable ética social. Para ello, usaba todas las técnicas que tenía a su disposición: desde la burla y la amenaza cuando se dirigía a los malos, hasta la alabanza y la admiración cuando se trataba de los buenos (Monique Nomo Ngamba, 2006).

La educación africana indígena era un proceso que duraba toda la vida. Era un permanente proceso de aprendizaje mediante el cual una persona avanzaba graduándose a través de etapas predeterminadas en la vida desde la cuna hasta la tumba. La educación es un viaje, en lugar de un trampolín inspirado hacia el éxito monetario. La educación en África no terminaba nunca. Se aprovecha cada momento para transmitir valores (Antonio Fernández, 2016). 
Fotografía n. ${ }^{4}$ : Cuentos y diversos relatos alrededor del fuego

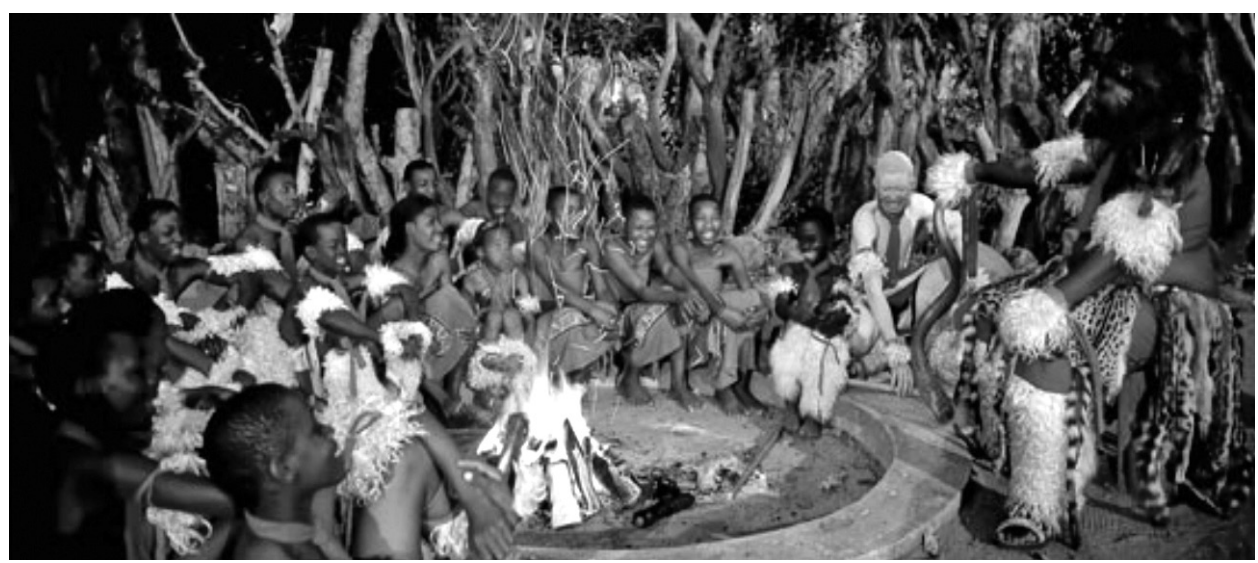

Fuente: Internet 2018.

\section{Principios pedagógicos y didácticos de la educación tradicional}

\section{I. Principios pedagógicos de la educación tradicional africana}

El etnógrafo Pierre Erny (1972, I977, I98I, I990, 200I) realizó muchos estudios sobre el niño africano, la educación en África subsahariana y el pensamiento africano sobre la educación. Observó durante sus investigaciones que las intervenciones de los adultos en la educación cotidiana se reducen al mínimo. «El niño se levanta libremente, solo, en medio de los suyos y especialmente de sus compañeros. Aprende por imitación, participando en la vida cotidiana de su grupo social. Realiza obras, participa en conversaciones y juegos, practica rituales. La participación de la educación en sí es a menudo insignificante. La educación se hace en la vida y por la vida» (Pierre Erny, I990: I80). No hay exámenes, ni pruebas cognitivas formales con diplomas.

Así, el primer principio pedagógico es el par observación-imitación. El fundamento educativo tradicional es aprender a observar para poder imitar. El niño debe imitar un modelo o un arquetipo. Debe observar y reproducir el prototipo. Su entorno social le proporciona los hitos que está obligado a seguir. Hay una ritualización en los actos de enseñanza/aprendizaje.

El segundo principio es el aprendizaje por experiencia o experimental. No existe un discurso sobre el objeto de aprendizaje. Por ejemplo, el joven aprende a cazar cazando. El niño aprende a nadar nadando. Igual para la pesca, se aprende realizando la actividad que se quiere adquirir. La niña aprende a cocinar cocinando.

El tercer principio es la autonomía. Permite que la fuerza interna que está dentro de cada individuo se revele a sí misma, mucho más que actuar sobre el niño para imponerle una forma desde el exterior. Debe adquirir un espíritu de responsabilidad ante la vida y ser capaz de desenvolverse. La educación tradicional se refiere a influencias inconscientes e informales. No se realiza en una institución de tipo escolar en un entorno organizado diariamente. 


\subsection{Elementos de comparación: principios didácticos de la educación tradicional}

Para P. Erny (1990) hay que distinguir respecto a la tradición científica europea que se basa en una observación cuidadosa y precisa. Describe al niño en todas las manifestaciones de su psique, su crecimiento, madurez, pureza, etc. Es sensible al comportamiento y busca saber cómo funcionan las cosas. Mientras que «la tradición africana también se basa en una observación de una agudeza implacable». Sin embargo, «no se detiene ahí» porque «a través de las apariencias, quiere conocer los significados, ir más allá de lo que aparece. Busca ser» (p. I4). Pero el problema agudo del continente africano es su permeabilidad a otras civilizaciones.

Surgen dos tipos diferentes de orientación y de espíritu entre el enfoque africano de la educación y la concepción occidental. En África la educación no está separada de la vida concreta. La práctica de esta educación también funciona como una especie de mayéutica en la que cada uno descubre sus propios talentos. Es el principio de emprendimiento. Y con la llegada de la escuela occidental, se ha creado una ruptura entre el vivir del niño y lo que aprende, cómo lo aprende y dónde aprende.

\subsection{La situación del niño africano después del contacto con otras culturas}

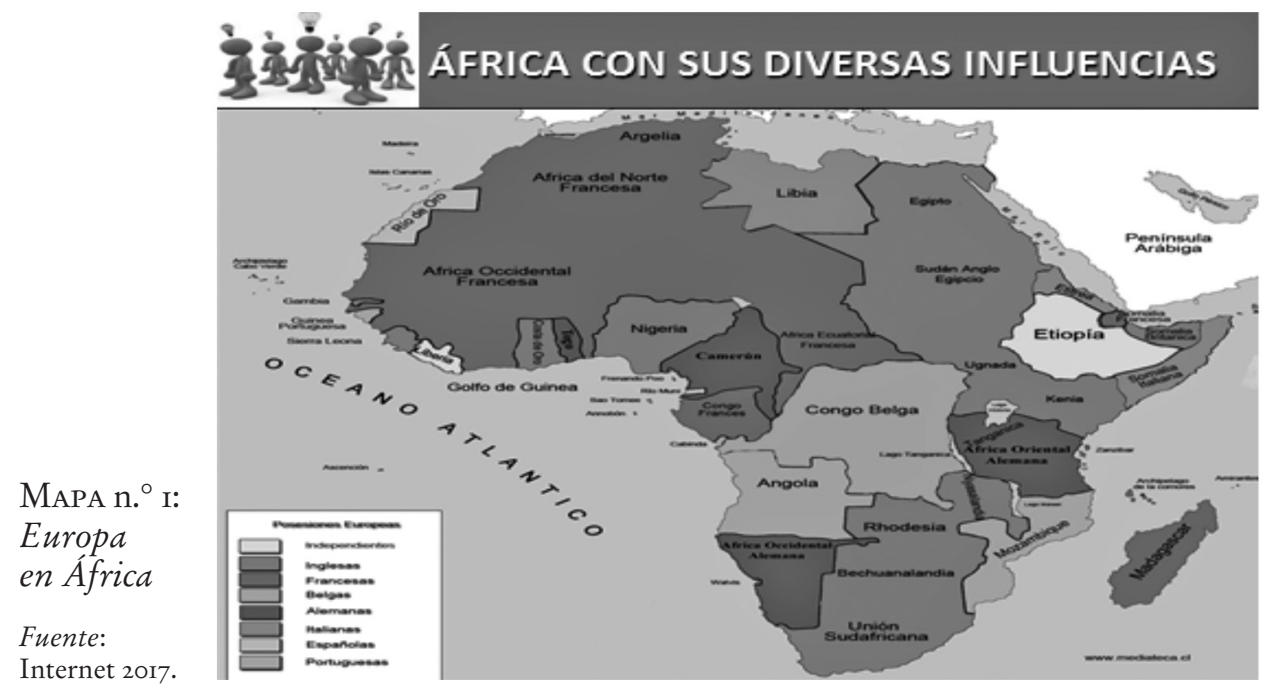

Cada país africano es una antigua colonia europea, excepto Etiopía. Son antiguas zonas de ocupación francesa, inglesa, portuguesa, alemana, italiana o española. Existen entonces en cada niño africano dos realidades educativas que compiten hoy en día en él.

\subsection{La yuxtaposición de dos concepciones: herencia de la colonización}

Muchos creen que se ha creado un ambiente educativo especial para el niño africano. La escuela occidental tal como se ha organizado en África tiene el efecto principal 
de vaciar al niño de sus raíces. Se trata de un entrenamiento artificial (Erny, 1977: 154). Por un lado, hay un desgarramiento entre la civilización ancestral global y los valores recibidos con ocasión de la formación institucional europea y, por otro lado, el «papel de la dominación del sistema social y cultural en África, en particular mediante la transferencia de sus propios patrones de análisis» (Fonkoué, 1985: 184). Los discursos coloniales traducen la transmisión de la ideología y del sistema de valores de las clases dominantes en Europa.

La segunda misión confiada a la escuela colonial era entonces la destrucción de los modos de vida y cultura endógenos tal como la adquisición de principios y formas de vida ajenos a la realidad socioeconómica nacional africana (Eyeang, 20II). Diversos escritos muestran que el niño africano está a horcajadas entre dos educaciones: la tradicional (ET) y la moderna (EO).

Para Erny (1977), y comparto su punto de vista, no hay razón para encerrar al joven africano en su entorno. Cualquier cosa que pueda abrirlo al exterior es buena y bienvenida. Conviene evitar que ingiera las nociones enciclopédicas del mundo entero antes de despertar en el alumno los rasgos múltiples de un universo familiar que puede observar y describir a partir de una experiencia vivida.

Y se llega a la conclusión de que hoy el «niño africano» no es uno, son dos. Orientarle exclusivamente en una dirección u otra es siempre mutilarle. Se puede pensar que, en la educación en África, deben tenerse en cuenta las contribuciones externas, sin perder de vista todos los valores de la cultura africana.

\section{Metodología de la investigación}

Nuestra investigación es cualitativa, basada en entrevistas semidirectivas e individuales. Su esencia es fenomenológica (Cook, 1986). La pregunta de partida que nos hacemos es: ¿Qué valor añadido tienen la educación tradicional y la educación europea boy en día?

Vamos a proceder a un análisis de contenido. El propósito es analizar las opiniones y representaciones a partir de discursos grabados (Bardin, 2013). La población entrevistada se compone de tres profesionales seleccionados por su experiencia personal con los dos tipos de educación y el mundo de la enseñanza:

- El primer sujeto (EA3) tiene 60 años. Es un profesor de escuela jubilado. Vive en Libreville y es abuelo. Recibió una educación totalmente tradicional. Conoció la época de la colonización.

- El segundo sujeto (EA2) tiene 40 años. Siempre ha vivido en el campo. Vive en Tchibanga (Nyanga, sur de Gabón). Es inspector pedagógico de lengua. Nació en el campo y llegó a la ciudad para cursar sus estudios.

- El tercer sujeto (EAI) tiene 30 años. Es doctor ingeniero en inteligencia artificial y vive en Francia. Nació en Francia, después de la independencia de Gabón. Siempre ha vivido en centros urbanos. Su educación se hizo fuera de África.

La meta es comparar los principios de la educación africana y los de la educación europea. La originalidad de las tres entrevistas que aparecen en este estudio nos parece ser la complementariedad de la información proporcionada por los sujetos entrevistados. 


\section{Presentación y análisis de los resultados}

Los resultados obtenidos de las entrevistas se organizan en torno a las orientaciones y los principios de la educación tradicional, por una parte. Por otra parte, presentamos las representaciones sobre la educación occidental.

\section{Tabla n. ${ }^{\circ}$ I: Las líneas principales de la educación tradicional}

\begin{tabular}{|c|c|}
\hline \multicolumn{2}{|c|}{ LAS LÍNEAS PRINCIPALES DE LA EDUCACIÓN TRADICIONAL } \\
\hline Ítems & Respuestas de los entrevistados \\
\hline \multirow{2}{*}{$\begin{array}{l}\text { I. El principio del modelo } \\
\text { El papel de los padres } \\
\text { y ancianos }\end{array}$} & $\begin{array}{l}\text { EA3: [...] //// El papel del niño en esta sociedad es que sigue los } \\
\text { pasos de los padres [padre o madre, tío o tía, tutor] (traducción } \\
\text { nuestra). }\end{array}$ \\
\hline & $\begin{array}{l}\text { EA2: Sí / }[\ldots][\ldots] \text { / el niño es: a veces es necesario ser } \\
\text { DISCIPLINADo / puedo tomarte un dominio / por ejemplo si los } \\
\text { padres eran religiosos ¿̇o es / el niño estaba obligado a ser } \\
\text { aunque no venga del corazón / [...] }\end{array}$ \\
\hline $\begin{array}{l}\text { 2. Los modos de } \\
\text { transmisión de los } \\
\text { saberes }\end{array}$ & $\begin{array}{l}\text { EAI: AH / [...] / Para mí educación tradicional se refiere a: } \\
\text { cuatro uh cuatro elementos básicos / ie uh :.: el aspecto social } \\
\text { / el aspecto político / el aspecto religioso y luego [...] / los } \\
\text { antepasados han codificado de hecho la sociedad de tal manera } \\
\text { que estos cuatro puntos pueden ser transmitidos a los niños / } \\
{[\ldots] \text { / }}\end{array}$ \\
\hline $\begin{array}{l}\text { 3. La apropiación del } \\
\text { sistema de creencias: } \\
\text { Momentos de } \\
\text { aprendizaje }\end{array}$ & $\begin{array}{l}\text { EAr: El niño debe por ejemplo euh :::: para hacer conexiones } \\
\text { con el clan con un clan diferente es comúnmente conocido } \\
\text { como matrimonio o alianza / [...] / } \\
\text { el niño también debe desarrollar el principio de poder } \\
\text { asegurando a los descendientes a nivel religioso / } \\
\text { Los «ancianos» también han codificado la sociedad de tal } \\
\text { manera que una serie de principios que están relacionados } \\
\text { con euh /// lo que llamaré el tótem }[. . .] \text { aproximadamente el } \\
\text { sistema de creencias }[\ldots . .] \text { puede ser transmitida [...]. }\end{array}$ \\
\hline $\begin{array}{l}\text { La tra } \\
\text { princi }\end{array}$ & $\begin{array}{l}\text { EAr: un conjunto de normas de valores como, por ejemplo, } \\
\text { el lenguaje, las prácticas cotidianas, uh, el respeto de los } \\
\text { «ancianos» frente a los demás / A nivel político / «Yo diré } \\
\text { que los viejos» han codificado la sociedad de nuevo para que } \\
\text { el niño por ejemplo / pueda transmitir y pueda reproducir un } \\
\text { conjunto de principios políticos que son importantes / }\end{array}$ \\
\hline $\begin{array}{l}\text { 4. Una educación } \\
\text { intuitiva }\end{array}$ & $\begin{array}{l}\text { EA3: La educación tradicional fue una educación intuitiva // } \\
{[\ldots] / /[\ldots] / / \text { No hubo ningún discurso sobre el aprendizaje // }}\end{array}$ \\
\hline $\begin{array}{l}\text { La «escuela para } \\
\text { la vida» }\end{array}$ & $\begin{array}{l}\text { Nosotros, por ejemplo, que somos del Ogooue [Río de I20o } \\
\text { km que cruza Gabón de este a oeste] / era necesario saber } \\
\text { nadar y remar // la primera cosa que hacíamos para enseñarnos } \\
\text { a nadar era echarnos en el agua / luchábamos y después de que } \\
\text { sabíamos nadar y terminó / fue como eso // }\end{array}$ \\
\hline
\end{tabular}



EUGÉNIE EYEANG

\begin{tabular}{|c|l|}
\hline \multicolumn{2}{|c|}{ LAS LÍNEAS PRINCIPALES DE LA EDUCACIÓN TRADICIONAL } \\
\hline \multicolumn{1}{|c|}{ Ítems } & \multicolumn{1}{|c|}{ Respuestas de los entrevistados } \\
\hline $\begin{array}{l}\text { Un aprendizaje práctico } \\
\text { por la experiencia }\end{array}$ & $\begin{array}{l}\text { Aprender a remar no se puede decir que es así como } \\
\text { mantenemos la paleta no! Usted ve cómo los demás mantienen } \\
\text { la paleta / Aprenda a limpiar la plantación Usted ve cómo } \\
\text { limpiar la plantación //// [...] Aprender a construir una red } \\
\text { [para la pesca] era la misma /// [...]// La educación tradicional } \\
\text { fue una educación para la vida // Ayudó al niño a hacerse cargo } \\
/ /[\ldots]\end{array}$ \\
\hline
\end{tabular}

Al analizar el discurso del sujeto EAI, se pueden apreciar las constantes de la educación tradicional africana habitual: el clan, el linaje, el tótem (sistema de creencias), etc. El uso recurrente de «viejo» o «antepasado» (cuatro veces en esta secuencia y doce veces en toda la entrevista) muestra que hay una referencia de la que los miembros no deben desviarse. Sin embargo, el sujeto EAI usa aquí el término «codificación». Es como si algo escrito existiera. Se trata de prácticas rutinarias «que el niño debe integrar». De las líneas principales de la educación tradicional, vamos a destacar los principios correspondientes en la tabla siguiente:

TABLA.$^{\circ}$ 2: Los principios de la educación tradicional

\begin{tabular}{|l|l|}
\hline \multicolumn{2}{|c|}{ LOS PRINCIPIOS PEDAGÓGICOS PRESENTES EN LA EDUCACIÓN TRADICIONAL } \\
\hline \multicolumn{1}{|c|}{ Ítems } & \multicolumn{1}{c|}{ Principios } \\
\hline $\begin{array}{l}\text { El principio del modelo } \\
\text { El papel de los padres, ancianos y ancestros }\end{array}$ & $\begin{array}{l}\text { Observación, Imitación, Reproducción } \\
\text { Sacralidad de los mayores } \\
\text { Referencia al pasado }\end{array}$ \\
\hline $\begin{array}{l}\text { Los modos de transmisión de los conoci- } \\
\text { mientos, principios y valores }\end{array}$ & $\begin{array}{l}\text { Transmisión unilateral, unidireccional, visión } \\
\text { única }\end{array}$ \\
\hline La apropiación del sistema de creencias & $\begin{array}{l}\text { Enfoque comunitario, Voluntario, Sin discu- } \\
\text { sión, Cosmogonía }\end{array}$ \\
\hline Una educación intuitiva & $\begin{array}{l}\text { Técnica de la observación, Comunicación no } \\
\text { verbal }\end{array}$ \\
\hline La «escuela para la vida» & Visión holística, visión global del hombre \\
\hline Un aprendizaje práctico y secreto & $\begin{array}{l}\text { Utilidad inmediata, Servicio a la comunidad } \\
\text { pero no compartido }\end{array}$ \\
\hline
\end{tabular}

La educación tradicional parece preparar al niño en el día a día. La referencia permanente al pasado da la impresión de no trabajar mucho para las perspectivas del futuro. El hombre está arraigado a su comunidad y vive por ella. Funciona a partir de un modelo, un arquetipo. Cada uno mira al patrón para poder ejecutar la tarea. Entonces, se aprende a observar, imitar y reproducir, tanto en el lenguaje como en las actitudes y acciones. El niño está sometido a las creencias y a la disciplina de los adultos. Hay muchos momentos de silencio, sin comunicación directa y explícita entre el niño y el adulto.

Tenemos la ilustración de esta realidad con el trabajo del hierro (Erny, 20or). Se trata de un niño que aprende el oficio de herrero observando a su padre, maestro herrero: 
Avant chaque exercice, l'observation de mon père au travail me donnait une idée des étapes de la tâche à effectuer. J'anticipais mentalement mes gestes. J'avais envie de comprendre le pourquoi des choses et d'affronter ce que je ne connaissais encore que par intuition. Mais au moment d'appliquer ce que déjà je pensais maîtriser, je butais sur des difficultés, surtout dans la coordination des gestes. Celles-ci n'étaient pas insurmontables, et j'étais ainsi poussé à reprendre le même exercice en modifiant mes conduites initiales. Mon père était pour moi un modèle très stimulant (Erny, 20or: 71-72).

El joven se desenvuelve solo para superar los obstáculos surgidos en su camino. Desarrolla cierta autonomía para lograrlo. Hay un diálogo interior que le permite repasar las etapas del trabajo del hierro, por la intuición. El aprendiz practica la autoevaluación porque es capaz de identificar los fallos en su práctica. Repite el ejercicio varias veces hasta la satisfacción personal.

La reproducción del patrimonio cultural aparece como el cemento de la sociedad tradicional, como se ha dicho anteriormente. El niño/el joven debe imitar lo que hacen sus padres, de manera fiel. No hay forma de cambiar las creencias. La crítica no se admite. El saber no se comparte. Es algo sagrado para los que lo preservan. Así es como se ha comparado a menudo al sabio africano con una biblioteca. Amadou Hampaté Bâ (I900-199I) nos dijo que «en África, cuando un anciano muere es como una biblioteca en llamas». Porque es depositario de un conocimiento meramente oral y no lo comparte con los demás.

Esta visión es diferente en la educación occidental cuya tendencia es la de poner al alcance del público una gran variedad de conocimientos.

\section{TABLA n. ${ }^{3}$ : La educación occidental según los entrevistados}

\section{LAS REPRESENTACIONES SOBRE LA EDUCACIÓN OCCIDENTAL}

\begin{tabular}{|c|r|}
\hline Ítems & Respuestas de los entrevistados \\
\hline
\end{tabular}

I. El papel de la escuela EAI: [...] //// ... alors que l'éducation l'école l'école c'est pour moi un module optionnel on peut aller à l'école ou ne pas aller à l'école / [...]. Mi [...] // L'école c'est c'est un module EN PLus qui vient justement compléter cette éducation traditionnelle

EAr: En Afrique oui / aujourd'hui aujourd'hui l'école / [...] / l'école c'est comme un ajout à cela ça vient compléter Euh [...] euh:::

2. El pensamiento de la $\quad$ EAr: [...] l'école va plutôt va plutôt donner va plutôt développer escuela occidental chez l'enfant le goût de la perfection le goût de la performance le goût de:: / [...].

EAI: l'école c'est une autre démarche c'est un autre concept / IL FAUT PERFECTIONNER LES CHOSES euh: / /

3. Una educación en constante evolución y abierta al cambio

EAr: la preuve c'est: c'est regarder euh le système le sytème de pensée ou philosohique occidental / Un phénomène en lui-même n'est jamais fini / Si si si aujourd'hui on te dit là c'est bleu

4. Una educación opuesta a la educación tradicional EAI: demain un autre courant de pensée ou bien une autre façon de voir cherchera et fera tout pour démontrer qu'en fait ce bleu // il peut être rouge il peut être: I Ce mécanisme en fait// on ne le retrouve pas dans l'éducation traditionnelle.

Fuente: Eyeang. 

EUGÉNIE EYEANG

Los entrevistados han podido destacar por sus experiencias individuales que la filosofía de la educación occidental es diferente de la tradicional.

TABLA n. ${ }^{\circ}$ : Los principios de la educación occidental

\begin{tabular}{|l|l|}
\hline \multicolumn{2}{|c|}{ Los principios pedagógicos presentes en la educación occidental } \\
\hline \multicolumn{1}{|c|}{ Ítems } & \multicolumn{1}{c|}{ Principios } \\
\hline La libertad de aprender & El principio de libre elección \\
\hline $\begin{array}{l}\text { Los modos de transmisión de los } \\
\text { conocimientos }\end{array}$ & Transmisión plural y multidimensional \\
\hline El sistema escolar & El principio de perfección y de performancia \\
\hline Una educación reflexiva & Mejoramiento permanente de lo existente \\
\hline Una educación abierta & Aceptación de la contradicción \\
\hline
\end{tabular}

Fuente: Eyeang.

\section{Discusión sobre la coexistencia de la educación tradicional y la occidental en los sistemas educativos africanos}

Las diferentes influencias que ha conocido África no han descartado completamente el tejido socioeducativo tradicional. La coexistencia de ambas filosofías merece una discusión en este trabajo en África.

TABLA n. ${ }^{\circ}$ : Comparación entre los principios de la educación en el siglo XXI y la ET

\begin{tabular}{|c|c|}
\hline $\begin{array}{l}\text { Principios de la educación en el siglo XXI } \\
\text { (García, 20I2) }\end{array}$ & $\begin{array}{c}\text { Principios de la educación tradicional } \\
\text { africana }\end{array}$ \\
\hline I. Ser un alumno de por vida & Escuela de la vida \\
\hline 2. Ser apasionado & Observación, imitación, reproducción \\
\hline 3. Estar dispuesto a asumir riesgos & Autonomía \\
\hline 4. Ser capaz de pensar de forma crítica & No hay sitio para la crítica \\
\hline $\begin{array}{l}\text { 5. Ser capaz de completar las cosas de modo } \\
\text { diferente }\end{array}$ & $\begin{array}{c}\text { Herencia del pasado: papel de los ancestros } \\
\text { y ancianos }\end{array}$ \\
\hline 6. Ser creativo & Imitación del modelo \\
\hline 7. Ser capaz de perseverar & Disciplina \\
\hline 8. Ser íntegro y respetarse a sí mismo & Responsabilidad, respeto \\
\hline 9. Tener coraje moral & Práctica, experiencia \\
\hline $\begin{array}{l}\text { Io. Ser capaz de usar el mundo que tiene en } \\
\text { torno suyo }\end{array}$ & Armonía con la naturaleza \\
\hline $\begin{array}{l}\text { II. Ser capaz de trabajar tanto de forma } \\
\text { independiente como con otras personas }\end{array}$ & Bajo la influencia de la comunidad \\
\hline I2. Hablar bien, leer bien, escribir bien & Enfoque oral \\
\hline
\end{tabular}

Fuente: Estudio. 
Al examinar esta tabla, conviene decir que hay aspectos convergentes y divergentes. El primer aspecto sobresaliente es la escuela para la vida. Es un concepto de la escuela tradicional (ET) transformado que muestra que aprender no es una etapa de nuestra vida, sino que es nuestra vida (García, 20I2). La oposición se manifiesta a nivel del uso de los conocimientos y del lugar que da la educación occidental (EO) a la creatividad y la movilización de todos los recursos a nuestra disposición. La postura voluntarista por una parte (EO) y la expectante por otra (ET) establecen otra diferencia. Se trata de provocar la realización de su destino de manera activa y no esperar las oportunidades de acción como si fueran trenes.

Se nota aquí como un deseo de integración de las posturas. El objetivo perseguido es decir como Javier García, 20I2:

Quiero estudiantes que aprendan a usar los recursos que tienen a su alrededor. Quiero que cuando lean o vean algo que les interese, lo persigan. Quiero que cuando tengan una idea, cojan un teléfono y llamen a alguien para hablar sobre ella, o que lean e investiguen, que se sienten y escriban. Cuando imagino a uno de mis estudiantes de adulto, pienso en una persona que es capaz de pensar y de hacer y seguir sus pasiones. Un adulto con la fortaleza para levantarse y hablar sobre sus pasiones, sus preocupaciones; [...] capaz de comprender y de hacerse comprender... [...] donde la creatividad, la pasión, el coraje y la perseverancia representen sus cualidades personales.

No es fácil tener dos tipos de educación. De hecho, en tal situación (Erny, I990: I79), «el niño debe aprender a jugar con un doble teclado cultural, repensar su forma de pensar, expresarse y compararse de acuerdo con las circunstancias y el entorno en el que se encuentra». La ET constituye la base de la personalidad del niño africano. Y la Eo le proporciona el complemento necesario para que su formación sea considerada como completa hoy.

Entre estos dos tipos de influencias la síntesis parece a primera vista imposible. Están animados por dos lógicas incompatibles, y uno prácticamente no tiene control sobre el otro. Se yuxtaponen sin realmente interactuar. Dentro del organismo psíquico hay dos sectores relativamente autónomos cuyas relaciones se rigen por lo que Roger Bastide ha llamado el «principio del corte». Según las circunstancias, «el individuo vive, piensa y reacciona de acuerdo con uno de los sistemas, poniendo el otro entre paréntesis, y esto, usualmente, con relativa facilidad, sin ningún rasgón u oposición dramática» (1977: 157).

También, Carl R. Rogers (I979: 20I) escribe que «un individuo está completamente abierto a las dos fuentes esenciales. Los datos proporcionados por lo que se siente dentro de uno mismo, y los datos por lo que se proporciona desde el mundo exterior». Por lo tanto, las capacidades intrínsecas de cada individuo permiten que el niño gabonés se adapte a las diferentes situaciones educativas a las que está expuesto.

La pedagogía moderna que complementa la educación tradicional en el niño africano no parece haber borrado la personalidad básica. Este último tiene ciertamente dificultades para asimilar los requisitos de la pedagogía occidental. Pues es el espíritu de imitación heredado de la educación consuetudinaria el que indudablemente justifica el rastreo mecánico en África negra de modelos elaborados para diferentes públicos. Sin embargo, hay que recordar que en la base la escuela aparece como un medio extranjero, totalmente diferente. Pasamos por la puerta de la escuela como si 


\section{Fotografía n. ${ }^{\circ}$ : Una educación jaspeada}

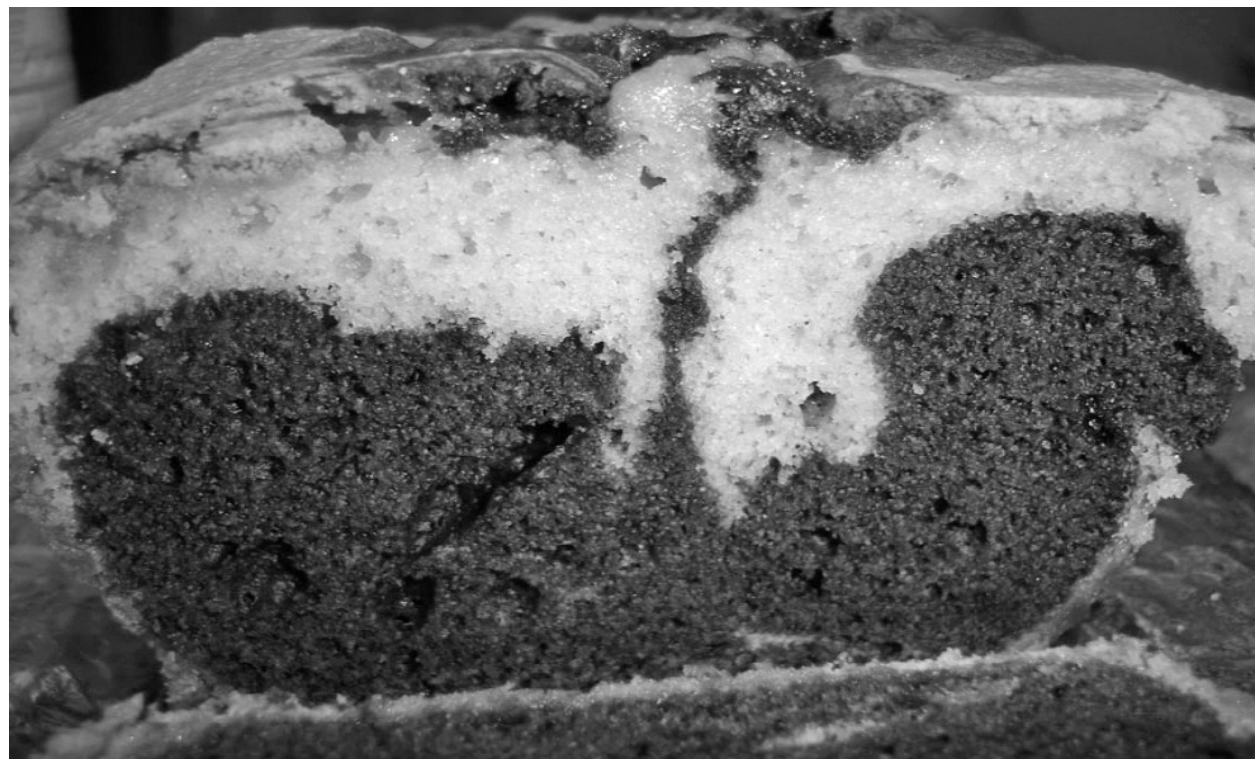

Fuente: Internet 2018 .

pasáramos una verdadera frontera, no solo frontera de conocimiento, sino frontera cultural, frontera de modos de ser, modos de sentir, modos de pensar.

Es cierto que «el conocimiento acostumbrado se empobrece, los lazos se distorsionan, las actitudes cambian, pero en su profunda sensibilidad el individuo permanece intensamente determinado» (Erny, I977: 157). Entonces, como señala esta mujer normanda senegalesa citada por P. Erny, «mi razón ha sido aclarada; pero mi cabeza es negra; pero mi sangre inexpugnable es pura, como el sol puro, preservado de todo contacto. Mi sangre ha permanecido pagana en mis venas civilizadas y revueltas y pacifica los sonidos de los tam-tams negros» (1990: 9).

De hecho, la contribución de la escuela -que abre las puertas de la civilización moderna- viene en segundo lugar en la formación del joven africano. Se localiza en un estrato relativamente superficial de personalidad. Pero hoy parece difícil negar su existencia, en la medida en que participa con la capa fundamental al futuro proyecto del joven africano. No se trata de introducir al maestro por el alumno, sino de provocar a los educandos para que se determinen a sí mismos. El contenido mismo de la enseñanza no es otra cosa que la situación del alumno tal como él mismo lo experimenta [...] con sus contradicciones, sus valores, sus juicios y el lenguaje con el que los expresa (Erny, I977: 82).

\section{Conclusión}

Educar no consiste en transmitir y memorizar contenidos. Tampoco consiste solo en adquirir una serie de conocimientos, los cuales corren el peligro de quedar obsoletos 
rápidamente. Se trata de ser capaz de utilizar e invertir todo lo que hemos aprendido en las situaciones-problemas que encontramos cada día.

En definitiva, se ha establecido un sistema para seguir los caminos trazados por los europeos. Pero, ¿cuál es la relevancia de esta actitud de los padres en el comportamiento de la población mundial? Pero ¿han perdido por completo sus raíces los que hoy son padres (con hijos de edad de aprender español, por ejemplo)? De manera similar, ¿ es posible que todos los ritos de iniciación hayan desaparecido por completo o que el sistema de creencias (basado en el culto de los antepasados) ya no exista?

Por lo tanto, debe reconocerse que ciertos puntos desaparecerán por las razones ya mencionadas, pero otros se resisten. Los esquemas de la ET -observación-imitación-reproducción- están tan integrados en los sujetos. De hecho, el conocimiento que se acumula ciertamente enriquece a los individuos, pero no siempre se pregunta si hay apropiación de los valores que acompañan estos conocimientos.

No obstante, para enseñar algo, es primordial comprenderlo de antemano. Por eso, los padres deben ser conscientes y congruentes en sus actos. Inculcar principios educativos significa promoverlos, asumirlos y cuestionarlos desde el ejemplo, es decir, no exigir algo que ellos no ponen en práctica. Aquella célebre frase de Albert Einstein, el físico teórico más brillante e influyente del siglo xx: "No intente convertirse en una persona de éxito, más bien intente convertirse en una persona de principios».

\section{Bibliografía}

Anderson, L. W. y Krathwohl, David Reading (eds.) (200I). A taxonomy for learning, teaching, and assessing: A revision of Bloom's taxonomy of educational objectives. New York: Longman.

Bardin, Laurence (2013). L'analyse de contenu. Paris: PUf.

Bloom, Benjamin S. y Krathwohl, David R. (1956). The classification of educational goals by a committee of college and university examiners. Longmans: New York: Longmans.

Boudon, R. y Bourricaud, F. (1983). Dictionnaire critique de la sociologie. Paris: Calmann-Lévy.

Canales, Manuel (2007). El enfoque comunitario. El desafío de incorporar a la comunidad en las intervenciones sociales de Víctor Martínez. Interamerican Journal of Psychology, ${ }_{4}(2)$, Porto Alegre.

Cook, Thomas D. (1986). Métodos cualitativos y cuantitativos en investigación evaluativa. Madrid: Ediciones Morata.

Echeverría, Javier (1995). Ciencia y valores. En Filosofía de la Ciencia (cap. III, pp. 67-79). Madrid: Akal.

Erny, Pierre (1972). L'enfant et son milieu en Afrique noire. Paris: Éditions Payot.

ERny, Pierre (1977). L'éducation dans les pays pauvres. Modèles et Propositions. Paris: L'Harmattan.

ERnY, Pierre (1990). L'enfant dans la pensée traditionnelle en Afrique noire. Paris: L'Harmattan.

ERny, Pierre (200I). Essai sur l'éducation en Afrique noire. Paris: L'Harmattan.

Eyeang, Eugénie (20II). El sistema educativo de Gabón, de la independencia a nuestros días (1960-20I0). Historia de la Educación. Revista Interuniversitaria, 30, 63-77. Salamanca: Ediciones Universidad Salamanca.

EyEANG, Eugénie (2015). L'importance des valeurs dans une société: Esquisse de la situation du Gabon. En E. Eyeang y F. R. Quentin De Mongaryas (dirs.) Les valeurs dans la société gabonaise. État des lieux, enjeux et perspectives (pp. 25-40). Libreville: Éditions odEM.

EYEANG, Eugénie (20I7). Los valores de la educación en los sistemas educativos africanos. En J. M. Hernández Díaz y E. Eyeang (eds.) Los valores en la educación de África. De ayer a boy (pp. 17-36). Salamanca: Ediciones Universidad Salamanca. 
García, Javier (20I2). Los principios educativos del siglo XXI. https://www.sintetia.com/ los-principios-educativos-del-siglo-xxi/. Consulta el I5.05.20I8.

García GarRIDO, José Luis (I99I). La educación comparada en una sociedad global. Revista Española de Educación Comparada, 3(1997), 6I-8I.

Kolb, David A. (1984). Experiential Learning. Experience as the Source of Learning and Development. Englewood Cliffs. NJ: Prentice-Hall.

Legendre, Renald (1993). Dictionnaire actuel de l'éducation. Montréal: Guérin, éditeur ltée.

Molina, Luzcarín et al. (2008). La importancia de formar en valores en la educación superior. www.actaodontologica.com/ediciones/2008/I/importancia_formar_valores_educacion_ superior.asp. Fundación Acta Odontológica Venezolana. RIF: J-30675328-I. ISSN: 000I-6365. Caracas. Venezuela.

Mougniotte, A. (1994). Eduquer à la démocratie. Paris: Cerf. Recherches morales, Positions.

Nomo Ngamba, Monique (2006). La narrativa negroafricana postcolonial en lenguas europeas y su crítica. Tonos. Revista Electrónica de Estudios Filológicos, II (julio). https://www. um.es/tonosdigital/znumis/estudios/r8-monique.htm.

SAnz MARTín, Pedro A. (20II). De la educación tradicional africana a la escuela actual en África subsahariana. TABANQUE. Revista Pedagógica, 24, 53-68. Universidad de Valladolid.

Schüтz, A. (1987). Le chercheur et le quotidien. Paris: Librairie des Méridiens, Klincksieck, Collection Société.

Sidibe, Fodé Moussa (200I). Transmission des savoirs: Le cas de la confrérie des chasseurs au Mali. Notre Librairie, I44, 69-75.

Stephenson, Joan et al. (comps.) (200I). Los valores en la educación. Madrid: Gedisa.

Valencia Infante, Elena (20I4). Las teorías de Freinet en la escuela. Madrid: Universidad Camilo José Cela.

Vinsonneau, Geneviève (1997). Culture et comportement. Paris: Armand Colin.

Vygotsky, Lev (1978). Mind in society: The development of higher psychological processes (ed. por M. Cole, V. John-Steiner, S. Scribner y E. Souberman). Cambridge, MA: Harvard University Press.

Wenger, Etienne (1998). Communities of Practice: Learning, Meaning, and Identity. Cambridge University Press. 
